\title{
Switching Stabilizability for Continuous-Time Uncertain Switched Linear Systems
}

\author{
Hai Lin, Member, IEEE, and Panos J. Antsaklis, Fellow, IEEE
}

\begin{abstract}
This paper investigates the switching stabilizability problem for a class of continuous-time switched linear systems with time-variant parametric uncertainties. First, a necessary and sufficient condition for the asymptotic stabilizability of such uncertain switched linear system is derived, under the assumption that the closed-loop switched system does not generate sliding motions. Then, an additional condition is introduced to exclude the possibility of unstable sliding motions. Finally, a necessary and sufficient for the asymptotic stabilizability of such continuous-time uncertain switched linear systems is presented. This result improves upon conditions found in the literature which are either sufficient only or necessary only.
\end{abstract}

Index Terms-Set-induced Lyapunov functions, stabilization, switched systems, uncertainty.

\section{INTRODUCTION}

A SWITCHED system is a dynamical system that consists of a finite number of subsystems described by differential or difference equations and a logical rule that orchestrates switching between these subsystems. Properties of this type of model have been studied for the past fifty years to consider engineering systems that contain relays and/or hysteresis. Due to its success in application and importance in theory, the last decade has seen increasing research activities in stability [8], [19], [22], controllability [34], [35], observability [1], [10], [14], stabilization [16], [25], [33], and switching optimal control [2], [39] of switched systems.

Among these research topics, stability and stabilization issues in switched systems have attracted most of the attention; see, e.g., the survey papers [8], [19], [20], [33], the recent books [18], [34], and the references cited therein. It is known that even when all the subsystems are unstable, the switched system may still generate convergent trajectories under certain class of switching signals. Then, a very interesting question is how "unstable" these subsystems can be while there still exists stabilizing switching signals. This is usually referred to in the literature as the switching stabilizability problem.

Early efforts on this issue focused on quadratic stabilization using a common quadratic Lyapunov function. For example, a quadratic stabilization switching law between two linear time

Manuscript received August 31, 2004; revised April 21, 2006. Recommended by Associate Editor A. Bemporad. This work was supported in part by the National Science Foundation under Grant NSF CCR01-13131.

H. Lin is with the Electrical and Computer Engineering Department, the National University of Singapore, Singapore 117576, Singapore. (e-mail: elelh@nus.edu.sg).

P. J. Antsaklis is with the the Electrical Engineering Department, the University of Notre Dame, Notre Dame, IN 46556 USA (e-mail: antsaklis.1@nd.edu)

Color versions of one or more of the figures in this paper are available online at http://ieeexplore.ieee.org.

Digital Object Identifier 10.1109/TAC.2007.894515 invariant (LTI) systems was considered in [37], where it was shown that the existence of a stable convex combination of the two subsystem matrices implies the existence of a state-dependent switching rule that stabilizes the switched system along a quadratic Lyapunov function. A generalization to more than two LTI subsystems was suggested in [27] by using a "min-projection strategy." In [12], it was shown that the stable convex combination condition is also necessary for the quadratic stabilizability of two mode switched LTI system. However, it is only sufficient for switched LTI systems with more than two modes. A necessary and sufficient condition for quadratic stabilizability of switched controller systems was derived in [31]. There are extensions of [37] to output-dependent switching and to the discrete-time case [19], [41]. For robust stabilization, a quadratic stabilizing switching law was designed for polytopic uncertain switched linear systems based on linear matrix inequality (LMI) techniques in [41]. All of these methods guarantee stability by using a common quadratic Lyapunov function, which is conservative in the sense that there are switched systems that can be asymptotically (or exponentially) stabilized without using a common quadratic Lyapunov function [14].

More recent efforts were based on multiple Lyapunov functions, especially piecewise quadratic Lyapunov functions, to construct stabilizing switching signals. For example, in [36], piecewise quadratic Lyapunov functions were employed for two mode switched LTI systems. Exponential stabilization for switched LTI systems was considered in [25], also based on piecewise quadratic Lyapunov functions, and the synthesis problem was formulated as a bilinear matrix inequality (BMI) problem. In [15], a probabilistic algorithm was proposed for the synthesis of an asymptotically stabilizing switching law for switched LTI systems along with a piecewise quadratic Lyapunov function. In [5], exponentially stabilizing switching laws were designed based on solving extended LQR optimal problems.

Related to the switching stabilization literature as described above, there is work on state or output feedback stabilization of switched systems or piecewise affine systems. For feedback stabilization, the switching signals are assumed to be given or restricted, while the continuous-variable control laws, in the form of state or output feedback, are designed to stabilize the switched systems under these given switching signals. Several classes of switching signals are considered in the literature, for example arbitrary switching [7], [11], slow switching [4], [13], restricted switching induced by partitions of the state space [6], [17], [29], [30] etc. The focus of this paper is on a typical switching stabilizability problem, where the switching signals are assumed to be free design variable, while no continuous-variable inputs are considered. 
Notice that the existing switching stabilizability conditions, which may be expressed as the feasibility of certain LMIs or BMIs, are sufficient only, except for certain cases of quadratic stabilization. An algebraic necessary condition for switching stabilizability of switched LTI was proposed in [32]. However, the proposed necessary condition was not sufficient except for the simplest case of one dimensional system. A necessary and sufficient condition for asymptotic stabilizability of second-order switched LTI systems was derived in [38] by detailed vector field analysis. However, it was not apparent how to extend the method to either higher dimensions or to the parametric uncertainty case.

This paper aims to derive a necessary and sufficient condition for asymptotic stabilizability of switched linear systems. In particular, it focuses on global asymptotic stabilizability (through static state feedback switching) for a class of switched linear systems with time-variant parametric uncertainties. The rest of the paper is organized as follows. In Section II, mathematical models for the uncertain switched linear system are described, and the asymptotic stabilizability problem is formulated. Section III generalizes the classical polytopic Lyapunov functions to polyhedral Lyapunov-like functions. Based on these Lyapunov-like functions, a stabilizing switching law is constructed and a global Lyapunov function is composed in Section IV, which guarantees asymptotic stability for the closed-loop switched linear system if no sliding motion occurs. The condition is proved to be also necessary for the existence of an asymptotically stabilizing switching control law without sliding motions. To handle sliding motions, an additional condition is introduced in Section V, which excludes the occurrence of unstable sliding motions in the closed-loop switched systems. It is shown that this additional condition is not conservative in the sense that it is automatically satisfied if the switched system can be asymptotically stabilized. Finally, concluding remarks are presented and future work is proposed.

\section{Notation}

In this paper, we use the letters $\mathcal{E}, \mathcal{P}, \mathcal{S} \cdots$ to denote sets. $\partial \mathcal{P}$ stands for the boundary of set $\mathcal{P}$, and $\operatorname{int}(\mathcal{P})$ its interior. A polyhedron in $\mathbb{R}^{n}$ is a (convex) set given by the intersection of a finite number of open and/or closed half-spaces in $\mathbb{R}^{n}$. A polytope is a closed and bounded (i.e., compact) polyhedron. A polytope (bounded polyhedral set) $\mathcal{P}$ will be presented either by a set of linear inequalities $\mathcal{P}=\left\{x: f_{i} x \leq g_{i}, i=1, \ldots, s\right\}$, and compactly by $\mathcal{P}=\{x: F x \leq g\}$, or by the dual representation in terms of the convex hull of its vertex set $\left\{x_{j}\right\}$, denoted by $\operatorname{Conv}\left\{x_{j}\right\}$. For any real $\lambda \geq 0$, the set $\lambda \mathcal{S}$ is defined as $\{x=\lambda y, y \in \mathcal{S}\}$. The term C-set stands for a convex and compact set containing the origin in its interior.

\section{PRoblem Formulation}

We consider a collection of continuous-time linear systems described by the differential equations with parametric uncertainties

$$
\dot{x}(t)=A_{q}(w) x(t), \quad t \in \mathbb{R}^{+}, q \in Q=\{1, \ldots, N\}
$$

where the state variable $x(t) \in \mathbb{R}^{n}$, the finite set $Q$ stands for the collection of discrete modes, and $\mathbb{R}^{+}$denotes non-negative real numbers. In particular, for all $q \in Q, A_{q}(w): \mathcal{W} \rightarrow$ $\mathbb{R}^{n \times n}$, and the entries of $A_{q}(w)$ are assumed to be continuous functions of $w \in \mathcal{W}$, where $\mathcal{W}$ is a given compact set in $\mathbb{R}^{v}$. The parametric uncertainty $w$ is assumed to be time-variant, and with unknown dependence on time $t$. Note that the origin $x_{e}=0$ is an equilibrium (maybe unstable) for the systems described in (1).

Combine the family of continuous-time uncertain linear systems (1) with a class of piecewise constant functions, $\sigma: \mathbb{R}^{+} \rightarrow$ $Q$, which serves as the switching signal between the collection of continuous-time systems (1). The continuous-time switched linear system can be described as

$$
\dot{x}(t)=A_{\sigma(t)}(w) x(t), \quad t \in \mathbb{R}^{+}
$$

and the switching signal is assumed to be generated by

$$
\sigma(t)=\delta(x(t))
$$

where $\delta: \mathbb{R}^{n} \rightarrow Q$. In other words, the discrete mode is determined by a form of static state feedback.

For this uncertain continuous-time switched system (2)-(3), an interesting question is: under what conditions it is possible to design switching laws such that the switched linear system is asymptotically stable. It is also desirable that the asymptotic stability property should be robust with respect to the parametric uncertainties. The main goal of this paper is to propose a necessary and sufficient condition for the existence of asymptotically stable switching laws for (2)-(3). The problem can be formulated as follows.

Problem: Given the continuous-time switched system (2)-(3), derive necessary and sufficient conditions, under which there exist switching control laws that make the closed-loop switched system globally asymptotically stable.

It is obvious that when one of the subsystems is stable, then this question has a trivial solution, i.e., just staying with this stable subsystem. Therefore, it is assumed that all the subsystems are unstable. The following assumption is made for these unstable subsystems (1).

Assumption: It is assumed that there exists a full row rank matrix $L_{q} \in \mathbb{R}^{m_{q} \times n}$, where $m_{q}<n$, such that the auxiliary system for the $q$-th subsystem (1)

$$
\dot{\xi}(t)=L_{q} A_{q}(w) R_{q} \xi(t), \quad t \in \mathbb{R}^{+}
$$

is asymptotically stable. Here $R_{q} \in \mathbb{R}^{n \times m_{q}}$ is a right inverse of $L_{q}$.

Intuitively, the previous assumption can be interpreted as considering a linear combination of the states of the original system (1) that evolves in an asymptotically stable manner in a subspace. The auxiliary system evolves in a lower dimensional state-space to which the original system can be projected for stability.

An interesting fact about the auxiliary system is that even when all parts of the state $x(t)$ are unbounded, there still may exist $L$ to satisfy the assumption. For example,

Example 1: Consider a continuous-time linear system

$$
\dot{x}(t)=\left[\begin{array}{cc}
0.5 & w \\
0 & 1
\end{array}\right] x(t)
$$


where the uncertain parameter $1 \leq w \leq 2$. The previous continuous-time system is obviously unstable. However, we may select $L=\left[\begin{array}{ll}1 & 0\end{array}\right]$ and $R=\left[\begin{array}{c}1 \\ -1\end{array}\right]$ to obtain

$$
L A(w) R=\left[\begin{array}{ll}
1 & 0
\end{array}\right]\left[\begin{array}{cc}
0.5 & w \\
0 & 1
\end{array}\right]\left[\begin{array}{c}
1 \\
-1
\end{array}\right]=0.5-w<0
$$

for all $w \in[1,2]$. Therefore, the auxiliary system

$$
\dot{\xi}(t)=(0.5-w) \xi(t)
$$

is asymptotically stable. Note that one may pick another $R$, for example, $R=\left[\begin{array}{c}1 \\ -a\end{array}\right]$ with $a>0.5$, to obtain a stable auxiliary
system

$$
\dot{\xi}(t)=(0.5-a w) \xi(t) .
$$

Based on the existence of a stable auxiliary system, the states $x$ contained in the range space of $R$ tend to converge to the null space of $L=\left[\begin{array}{ll}1 & 0\end{array}\right]$. This partial convergence property of the original system is captured by a polyhedral Lyapunov-like function that is developed in the next section.

If the subsystem is LTI, i.e., (1) becomes $\dot{x}(t)=A_{q} x(t)$, which corresponds to the uncertain parameter set $\mathcal{W}$ in (1) being a singleton, then it can be shown that there always exist $L_{q}$ and $R_{q}$ satisfying the above assumption, except when $A_{q}=\lambda I_{n}$ for some positive real $\lambda>0$. Here, $I_{n}$ is the identity matrix of dimension $n$. The proof of this claim is quite straightforward using the structure of the Jordan canonical form of $A_{q}$.

For the case that $A_{q}=\lambda I_{n}, \lambda \in \mathbb{R}^{+}$, there does not exist any stable auxiliary system. If we look at the phase plane of this subsystem, $\dot{x}(t)=\lambda I_{n} x(t)$, all the field vectors point to infinity along the radial directions. Intuitively speaking, the dynamics are explosive and do nothing but drive all the states to infinity, which we would like to avoid since no contribution is made to the stabilization purpose of a switched system. For this case, one may simply remove these subsystems with the dynamics of $\dot{x}(t)=\lambda I_{n} x(t)$.

For the parametric uncertainty case, $\dot{x}(t)=A_{q}(w) x(t)$, it will be shown in Section IV-B by contradiction that if the switched linear systems is stabilizable then there always exist $L_{q}$ and $R_{q}$ satisfying the aforementioned assumption. Therefore, the assumption on the existence of $L_{q}$ and $R_{q}$ does not cause loss of generality.

\section{PolyhedRal LyAPUnOV-Like FunCtions}

\section{A. Polytopic Lyapunov Functions}

Following the discussion in the previous section, each subsystem has an asymptotically stable auxiliary system

$$
\dot{\xi}(t)=L_{q} A_{q}(w) R_{q} \xi(t)
$$

in a lower dimensional $\mathbb{R}^{m_{q}}$. In this section, the discussion is restricted to a single subsystem, i.e., $q$ is fixed.

According to the converse Lyapunov theorem in [21], [23] for linear time-variant systems, the asymptotic stability of the auxiliary system implies the existence of a polytopic Lyapunov function, denoted as $\Phi_{q}(\xi)$. Following the notation in [3], [21], the polytopic Lyapunov function $\Phi_{q}(\xi)$ can be represented as

$$
\Phi_{q}(\xi)=\max _{1 \leq i \leq s_{q}}\left\{f_{i} \xi\right\}
$$

where $f_{i} \in \mathbb{R}^{1 \times m_{q}}$ is a nonzero row vector and $s_{q}$ is an integer greater than $m_{q}$. In addition, $\Phi_{q}(\xi)$ has the following properties [3]: $\Phi_{q}(\xi) \geq 0, \Phi_{q}(\xi)=0 \Leftrightarrow \xi=0$; for $\mu>0, \Phi_{q}(\mu \xi)=$ $\mu \Phi_{q}(\xi)$; and $\Phi_{q}\left(\xi_{1}+\xi_{2}\right) \leq \Phi_{q}\left(\xi_{1}\right)+\Phi_{q}\left(\xi_{2}\right), \forall \xi_{1}, \xi_{2} \in \mathbb{R}^{m_{q}}$. $\Phi_{q}(\xi)$ is continuous and piecewise linear. In fact, it defines a distance of $\xi$ from the origin which is linear in any direction.

Consider the following closed set:

$$
\begin{aligned}
\mathcal{P}_{q} & =\left\{\xi \in \mathbb{R}^{m_{q}}: \Phi_{q}(\xi) \leq 1\right\} \\
& =\left\{\xi \in \mathbb{R}^{m_{q}}: f_{i} \xi \leq 1, \forall 1 \leq i \leq s_{q}\right\} .
\end{aligned}
$$

It is known from [3] that the previous set $\mathcal{P}_{q}$ is a bounded polyhedral set with the origin in its interior, so it is a polyhedral C-set. If one takes $F_{q} \in \mathbb{R}^{s_{q} \times m_{q}}$ to be the matrix with $f_{i} \in$ $\mathbb{R}^{1 \times m_{q}}$ as its $i$ th row vector, then $\mathcal{P}_{q}$ can be represented as

$$
\mathcal{P}_{q}=\left\{\xi \in \mathbb{R}^{m_{q}}: F_{q} \xi \leq \overline{1}\right\}
$$

where $\overline{1}$ stands for a column vector in $\mathbb{R}^{m_{q}}$ with all elements being 1 and " $\leq$ " is component-wise.

It is worth pointing out that the polytopic Lyapunov function $\Phi_{q}(\xi)$ is the Minkowski function of $\mathcal{P}_{q}$ [3], i.e.,

$$
\Phi_{q}(\xi)=\inf \left\{\mu>0: \xi \in \mu \mathcal{P}_{q}\right\} .
$$

Therefore, the polytopic Lyapunov function $\Phi_{q}(\xi)$ is induced from a polyhedral C-set, so it is also called a set-induced Lyapunov function.

If the polyhedral C-set $\mathcal{P}_{q}$ is also 0 -symmetric, i.e., $x \in \mathcal{P}_{q}$ implies $-x \in \mathcal{P}_{q}$ as well, then the induced polytopic Lyapunov function $\Phi_{q}(\xi)$ can be expressed as

$$
\Phi_{q}(\xi)=\left\|F_{q} \xi\right\|_{\infty}=\max _{1 \leq i \leq s_{q}}\left\{\left|f_{i} \xi\right|\right\}
$$

where $\|\cdot\|_{\infty}$ stands for the infinity vector norm.

It is interesting that the results in [21] not only imply the existence of a polytopic Lyapunov function for an asymptotically stable auxiliary system, but also the Lyapunov function can always be selected to be of the above weighted $\infty$-norm form. Hence, the set $\mathcal{P}_{q}$ can always be selected as 0 -symmetric.

Several methods for automated construction of the polyhedral Lyapunov function have been proposed in the literature. Early results include [9], where the Lyapunov function construction was reduced to the design of a balanced polytope satisfying some invariance properties. An alternative approach was given by Molchanov and Pyatnitskiy in [23], where algebraic stability conditions based on weighted infinity norms were proposed. A linear programming based method for solving these conditions was given by Polański in [28]. Recently, in [40], Yfoulis and Shorten proposed a numerical approach, called ray-griding, to calculate polyhedral Lyapunov functions based on uniform partitions of the state-space in terms of ray directions. 


\section{B. Extension to Polyhedral Lyapunov-Like Functions}

Notice that all the derivations so far are restricted to the stable auxiliary system (4), which evolves in a lower dimensional state space $\mathbb{R}^{m_{q}}$. In the following, we will consider the original unstable subsystem (1) in $\mathbb{R}^{n}$. Since it is unstable, there does not exist a Lyapunov function in the classical sense. However, as illustrated in Example 1, the existence of a stable auxiliary system implies that some linear combinations of the states converge within certain subspaces. This kind of partial convergence property is captured by the existence of a polyhedral Lyapunov-like function for (1). The main aim of this section is to transform the polyhedral Lyapunov function $\Phi_{q}(\xi)$ of the auxiliary system (4) into a polyhedral Lyapunov-like function for the original subsystem (1) in $\mathbb{R}^{n}$.

For this, we need to introduce the Euler Approximate System (EAS) for the auxiliary system (4)

$$
\xi[k+1]=L_{q}\left[I+\tau A_{q}(w)\right] R_{q} \xi[k], \quad k \in \mathbb{Z}^{+}
$$

where $\tau$ is a positive scalar.

In [3], Blanchini exploited the connection between the continuous-time system (4) and its corresponding discrete-time EAS (7), which is based on the concept of a contractive set for (7).

Definition 1: Given a positive scalar $\lambda, 0<\lambda<1$, a set $\mathcal{P}$ is said to be $\lambda$-contractive with respect to the discrete-time EAS (7), if, for any $\xi \in \mathcal{P}$, all the possible next step states through the transition (7) are contained in the set $\lambda \mathcal{P}$. That is

$$
L_{q}\left[I+\tau A_{q}(w)\right] R_{q} \xi \in \lambda \mathcal{P}
$$

holds for any $\xi \in \mathcal{P}, w \in \mathcal{W}$.

It is shown in [3], that for an asymptotically stable system (4), there always exist a positive scalar $\bar{\tau}>0$ and a positive constant $\lambda<1$ such that, for all $0<\tau \leq \bar{\tau}$, the level set $\mathcal{P}_{q}=$ $\left\{\xi: F_{q} \xi \leq \overline{1}\right\}$ in (5) is $\lambda$-contractive for the corresponding discrete-time EAS (7).

Therefore, for the auxiliary system (4), we obtain that

$$
F_{q} L_{q}\left[I+\tau A_{q}(w)\right] R_{q} \xi \leq \lambda \overline{1}
$$

holds for all $\xi \in \mathcal{P}_{q}=\left\{\xi: F_{q} \xi \leq \overline{1}\right\}$, for all $w \in \mathcal{W}$ and for $\tau$ small enough.

For all the states $x$ contained in the range space of $R_{q}$, one may represent $x=R_{q} \xi$. Note that the range space of $R_{q}$, denoted as image $\left(R_{q}\right)$, is a linear subspace of $\mathbb{R}^{n}$. Hence, for all $x \in\left\{x: F_{q} L_{q} x \leq \overline{1}\right\} \bigcap \operatorname{image}\left(R_{q}\right)$

$$
F_{q} L_{q}\left[I+\tau A_{q}(w)\right] x \leq \lambda \overline{1}
$$

holds for all $w \in \mathcal{W}$ and for all $0<\tau \leq \bar{\tau}$. As it has been pointed out in Example 1, the matrix $R_{q}$ that satisfies the assumption (4) and the corresponding EAS (7) admits $\mathcal{P}_{q}$ as a $\lambda$-contractive set, is not unique for a given $L_{q}$. Denote the union of the range space of all these $R_{q}$ as

$$
\mathcal{M}_{q}=\bigcup_{R_{q}} \operatorname{image}\left(R_{q}\right)
$$

and for all states $x \in\left\{x: F_{q} L_{q} x \leq \overline{1}\right\} \bigcap \mathcal{M}_{q}$

$$
F_{q} L_{q}\left[I+\tau A_{q}(w)\right] x \leq \lambda \overline{1}
$$

for all $w \in \mathcal{W}$ and for all $0<\tau \leq \bar{\tau}$.

Note that the discrete-time system

$$
x[k+1]=\left[I+\tau A_{q}(w)\right] x[k]
$$

is the corresponding EAS for the original subsystem (1). Let us denote the (convex but unbounded) polyhedral set $\mathcal{S}_{q}=\{x$ : $\left.F_{q} L_{q} x \leq \overline{1}\right\}$. One remarkable feature of the polyhedron $\mathcal{S}_{q}$ is that it is $\lambda$-contractive for the EAS (9) along $\mathcal{M}_{q}$. Hence, the set $\mathcal{S}_{q}$ is called partial contractive along $\mathcal{M}_{q}$. This is a generalization of the usual contractive set definition. ${ }^{1}$

In the following, it is shown that the existence of such partial contractive set $\mathcal{S}_{q}$ for the EAS (9) implies a polyhedral Lyapunov-like function, defined below, for the continuous-time subsystem (1).

Denote $F_{q} L_{q} \in \mathbb{R}^{s_{q} \times n}$ as $H_{q}$, and $h_{i}$ as the $i$ th row vector of $H_{q}$. Then, the polyhedral Lyapunov-like function candidate from the polyhedron $\mathcal{S}_{q}$ can be defined as

$$
\Psi_{q}(x)=\max _{1 \leq i \leq s_{q}}\left\{h_{i} x, 0\right\}
$$

Note that $\Psi_{q}(x) \geq 0$ by definition, and that $\Psi_{q}(x)$ is continuous and piecewise linear for $x$, and

$$
\mathcal{S}_{q}=\left\{x: F_{q} L_{q} x \leq \overline{1}\right\}=\left\{x: \Psi_{q}(x) \leq 1\right\}
$$

by definition. However, $\Psi_{q}(x)=0$ does not imply that $x=0$. In fact, for all $x$ contained in the convex cone

$$
\mathcal{C}_{q}=\left\{x: H_{q} x \leq \overline{0}\right\}
$$

we have $\Psi_{q}(x)=0$. This is one of the main differences from the classical Lyapunov function, so we call $\Psi_{q}(x)$ a Lyapunov-like function. The above Lyapunov-like function represents a nontrivial generalization of the classical polytopic Lyapunov functions.

Since the set-induced function $\Phi_{q}$ is continuous but not continuously differentiable [23], so is $\Psi_{q}$, we consider the (upper right) Dini derivative of $\Psi_{q}$ along the trajectory of the continuous-time system (1), which is defined as

$$
\mathcal{D}^{+} \Psi_{q}(x(t))=\lim \sup _{\tau \rightarrow 0^{+}} \frac{\Psi_{q}(x(t+\tau))-\Psi_{q}(x(t))}{\tau} .
$$

It was shown in [3] that the Dini derivative of $\Psi_{q}$ at the time instant $t$, for $x(t)=x$, and $w(t)=w$, can be calculated as

$$
\mathcal{D}^{+} \Psi_{q}(x(t))=\lim \sup _{\tau \rightarrow 0^{+}} \frac{\Psi_{q}\left(x+\tau A_{q}(w) x\right)-\Psi_{q}(x)}{\tau} .
$$

In the sequel, we will show that the Dini derivative of $\Psi_{q}$ is negative for all $x$ contained in $\mathcal{M}_{q}$ and outside the cone $\mathcal{C}_{q}$. The following property of the partial contractive sets for EAS (9) is

${ }^{1}$ If the set $\mathcal{M}_{q}$ is the whole state space $\mathbb{R}^{n}$, then one obtains the usual contractiveness. 
essential to prove that the Dini derivative of $\Psi_{q}$ is negative along the trajectory of (1).

Lemma 1: If $\mathcal{S}$ is a partial $\lambda$-contractive set for the EAS (9) along $\mathcal{M}_{q}$, then $\mu \mathcal{S}$ is so for all $\mu>0$.

The next lemma shows that the partial contractiveness of the polyhedral set $\mathcal{S}_{q}$ for the EAS (9) implies the negativeness of the Dini derivative of $\Psi_{q}$ for (1).

Lemma 2: If there exist scalars $0<\lambda<1$ and $\bar{\tau}>0$, such that the polyhedral set $\mathcal{S}_{q}=\left\{x: H_{q} x \leq \overline{1}\right\}$ is a partial $\lambda$-contractive set for the EAS (9) along $\mathcal{M}_{q}$ for all $0<\tau<\bar{\tau}$, then the Dini derivative $\mathcal{D}^{+} \Psi_{q}(x(t))$ for all $x(t)$ contained in $\mathcal{M}_{q}$ and outside the cone $\mathcal{C}_{q}$ is negative along the trajectory of the continuous-time system (1).

The previous two lemmas are direct extensions of the results in [3], and the proofs are omitted due to space limitation.

Notice that the intersection of $\mathcal{M}_{q}$ and $\mathcal{C}_{q}$ only contains the origin. This is because

$$
\begin{aligned}
\mathcal{M}_{q} \cap \mathcal{C}_{q} & =\left\{x=R_{q} \xi: F_{q} L_{q} R_{q} \xi \leq 0, \xi \in \mathbb{R}^{m_{q}}\right\} \\
& =\left\{x=R_{q} \xi: F_{q} \xi \leq 0, \xi \in \mathbb{R}^{m_{q}}\right\}
\end{aligned}
$$

where the set $\left\{\xi: F_{q} \xi \leq 0\right\}$ only contains $\xi=0$. This is due to the positiveness of $\Phi_{q}(\bar{\xi})$. Therefore, the condition "outside the cone $\mathcal{C}_{q}$ " is automatically satisfied for non-zero $x$ contained in $\mathcal{M}_{q}$.

The previous two lemmas can be seen as generalizations of the corresponding results in [3] to the case of unstable uncertain systems. Here, the negativeness of the Dini derivative is only guaranteed for part of the states, i.e., $x(t)$ contained in $\mathcal{M}_{q}$. A state trajectory starting in such regions may leave these regions, and the polyhedral Lyapunov-like function may then increase its value. Hence, from a single polyhedral Lyapunov-like function, one could not say much about the global stability of a switched system. However, if one patches these multiple polyhedral Lyapunov-like functions together in such a way that the union of these $\mathcal{M}_{q}$ is the whole $\mathbb{R}^{n}$ and the intersection of $\mathcal{C}_{q}$ is just the origin, then it is possible to deduce the switched system stability. This is exactly the main idea that will be employed in the sequel of this paper. Before the presentation of the main results, let us first take a look at a simple example to gain insight into the partial contractiveness of the polyhedral Lyapunov-like function.

Example 2: Consider a continuous-time linear system

$$
\dot{x}(t)=\left[\begin{array}{cc}
0 & 10+w \\
0 & 0
\end{array}\right] x(t)
$$

where $w \in[-1,0]$. It is not asymptotically stable, but we may select $L=\left[\begin{array}{ll}-1 & 1\end{array}\right]$ and parameterize $R$ as $R=\left[\begin{array}{c}r-1 \\ r\end{array}\right]$, we obtain

$$
\begin{aligned}
L A(w) R & =\left[\begin{array}{ll}
-1 & 1
\end{array}\right]\left[\begin{array}{cc}
0 & 10+w \\
0 & 0
\end{array}\right]\left[\begin{array}{c}
r-1 \\
r
\end{array}\right] \\
& =-(10+w) r .
\end{aligned}
$$

It is easy to verify that $L R=1$ and the auxiliary system

$$
\dot{\xi}(t)=-(10+w) r \xi(t)
$$

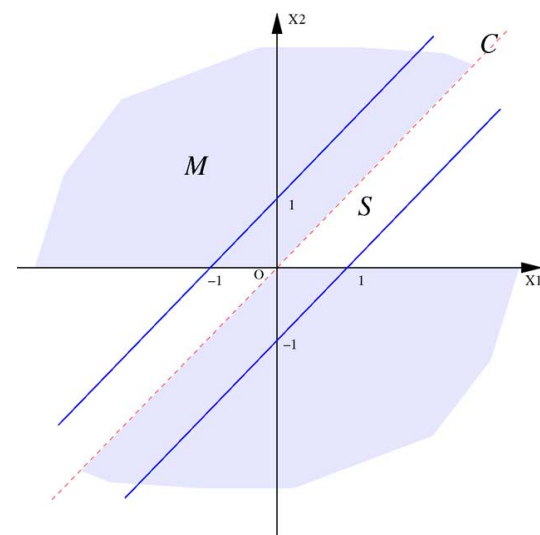

Fig. 1. Illustration of $\mathcal{S}$ and $\mathcal{M}$ for Example 2.

is asymptotically stable when $r>0$. All these stable auxiliary systems admit $\Phi(\xi)=|\xi|$ as their polytopic Lyapunov function, and the interval $\mathcal{P}=\{\xi:-1 \leq \xi \leq 1\}$ is a contractive set for the EASs of these auxiliary systems. In this example, the matrix $F=\left[\begin{array}{c}1 \\ -1\end{array}\right]$, and
\[ F L=\left[\begin{array}{c}1 \\ -1\end{array}\right]\left[\begin{array}{ll}-1 & 1\end{array}\right]=\left[\begin{array}{cc}-1 & 1 \\ 1 & -1\end{array}\right] . \]

Therefore, we obtain the polyhedral set $\mathcal{S}$ in $\mathbb{R}^{2}$

$$
\begin{aligned}
\mathcal{S} & =\{x: F L x \leq \overline{1}\} \\
& =\left\{x \in \mathbb{R}^{2}:\left[\begin{array}{cc}
-1 & 1 \\
1 & -1
\end{array}\right] x \leq\left[\begin{array}{l}
1 \\
1
\end{array}\right]\right\}
\end{aligned}
$$

from which a polyhedral Lyapunov-like function $\Psi(x)$ can be defined as

$$
\Psi(x)=\max \left\{\left[\begin{array}{ll}
-1 & 1
\end{array}\right] x,\left[\begin{array}{ll}
1 & -1
\end{array}\right] x, 0\right\} .
$$

Finally, we calculate the set $\mathcal{M}$ defined in (8) as

$$
\mathcal{M}=\bigcup_{r>0} \text { image }\left[\begin{array}{c}
r-1 \\
r
\end{array}\right]
$$

which is illustrated in Fig. 1. The level set of $\Psi(x)$, i.e., $\mathcal{S}=$ $\{x: \Psi(x)=1\}$, is also plotted in Fig. 1 .

To illustrate the negativeness of the Dini derivative of $\Psi(x)$, we select $x_{0}=\left[\begin{array}{c}2 \\ -1\end{array}\right]$. Note that $x_{0} \in \mathcal{M}$. In fact, $x_{0}$ is contained in the range space of $\left[\begin{array}{c}r-1 \\ r\end{array}\right]$ for $r=1 / 3$. For the state
$x_{0}$

$$
\begin{aligned}
\Psi\left(x_{0}\right) & =\max \left\{\left[\begin{array}{ll}
-1 & 1
\end{array}\right] x_{0},\left[\begin{array}{ll}
1 & -1
\end{array}\right] x_{0}, 0\right\} \\
& =\max \{-3,3,0\}=3 .
\end{aligned}
$$

The Dini derivative of $\Psi(x)$ at $x_{0}$ is

$$
\mathcal{D}^{+} \Psi\left(x_{0}\right)=\max _{w}\left[\begin{array}{ll}
1 & -1
\end{array}\right] A(w) x_{0}=-10<0
$$

Note that $\Psi\left(x_{0}\right)=\left[\begin{array}{ll}1 & -1\end{array}\right] x_{0}$. 
Note that a single subsystem could have more than one polyhedral Lyapunov-like functions with different selections of $L_{q}$ and/or $\Phi_{q}(\xi)$. This provides flexibility to assign several different polyhedral Lyapunov-like functions to the same single subsystem, and to treat them as different "new" modes for the switching stabilization. This argument makes it reasonable to only consider the case where $L_{q}$ is a row vector, i.e., $L_{q} \in \mathbb{R}^{1 \times n}$. When $L_{q}$ is a row vector, the auxiliary system becomes a scalar systems and makes the computation of $L_{q}$ and $R_{q}$ very simple, which can to be solved by linear programming. This is further discussed in the conclusion part of this paper.

\section{MAin Results}

In this section, we will derive a necessary and sufficient condition for the asymptotic stabilizability of the switched linear system (2)-(3) under the assumption that the closed-loop system does not generate sliding motions. In other words, we assume that there are only finite switchings within any finite time interval. Once sliding motion occurs, which is easy to detect by vector field analysis along switching surfaces, additional care should be exercised, and this will be dealt with in Section V.

The basic idea here is to patch together the polyhedral Lyapunov-like functions developed in the previous section. The way to patch them together is through the intersection of their level sets, i.e.,

$$
\mathcal{S}=\bigcap_{q \in Q} \mathcal{S}_{q}=\bigcap_{q \in Q}\left\{x \in \mathbb{R}^{n}: \Psi_{q}(x) \leq 1\right\}
$$

The set $\mathcal{S}$ is a finite intersection of polyhedral sets, so $\mathcal{S}$ is a polyhedron as well. In addition, $\mathcal{S}$ is nonempty, since every set $\mathcal{S}_{q}$ contains the origin in its interior. It is assumed that $\mathcal{S}$ is bounded $\operatorname{too}^{2}$. So $\mathcal{S}$ is a polyhedral C-set. Therefore, the Minkowski function of $\mathcal{S}$

$$
V(x)=\inf \{\mu>0: x \in \mu \mathcal{S}\}
$$

is non-negative, continuous, convex and piecewise linear and measures the distance of $x$ from the boundary of $\mathcal{S}$ as an inner product.

Let us introduce some notation for the polyhedral C-set $\mathcal{S}$. First, $\operatorname{vert}(\mathcal{S})=\left\{v_{1}, v_{2}, \ldots, v_{e}\right\}$ stands for its finite vertices, while $\operatorname{face}(\mathcal{S})=\left\{F_{1}, F_{2}, \ldots, F_{M}\right\}$ denotes its facets. The hyperplane that corresponds to the $k$ th facet $F_{k}$ is defined by

$$
\mathrm{H}_{k}=\left\{x \in \mathbb{R}^{n}: f_{k} x=1\right\}
$$

where $f_{k} \in \mathbb{R}^{1 \times n}$ is the corresponding gradient vector of facet $F_{k}$. The set of vertices of $F_{k}$ can be found as $\operatorname{vert}\left(F_{k}\right)=$ $\operatorname{vert}(\mathcal{S}) \cap F_{k}$. We denote the cone generated by the vertices of $F_{k}$ by cone $\left(F_{k}\right)$, and

$$
\begin{aligned}
& \operatorname{cone}\left(F_{k}\right) \\
& =\left\{x \in \mathbb{R}^{n}: x=\sum_{i} \alpha_{i} v_{k_{i}}, \alpha_{i} \geq 0, v_{k_{i}} \in \operatorname{vert}\left(F_{k}\right)\right\} .
\end{aligned}
$$

${ }^{2}$ The necessary and sufficient condition for the intersection of $\mathcal{S}_{q}$ to be bounded is given in Section IV-A.

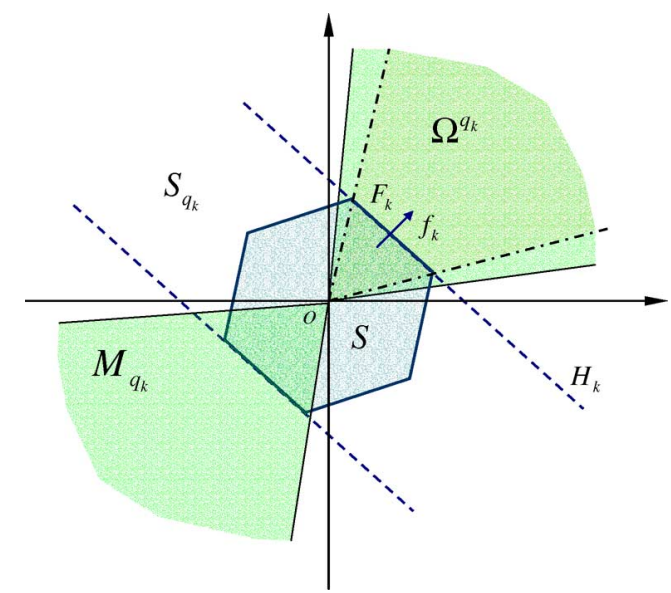

Fig. 2. Illustration of facet $F_{k}$, hyperplane $H_{k}$ and cone cone $\left(F_{k}\right)\left(=\Omega^{q_{k}}\right)$.

Note that

$$
\bigcup_{k=1, \ldots, M} \operatorname{cone}\left(F_{k}\right)=\mathbb{R}^{n} .
$$

Hence, we obtain a conic partition of the whole state-space $\mathbb{R}^{n}$.

For each facet of $\mathcal{S}, F_{k}$, there exists at least one mode $q$ such that $V(x)=\Psi_{q}(x)$. This is simply because of the fact that $\mathcal{S}$ is the intersection of $\mathcal{S}_{q}$ for $q \in Q$, and both $V(x)$ and $\Psi_{q}(x)$ are the measure of the distance of $x$ from the boundary of $\mathcal{S}$ (the intersected boundaries of $\mathcal{S}_{q}$ ). Collect all such modes $q$ such that $V(x)=\Psi_{q}(x)$, and call them feasible modes for cone $\left(F_{k}\right)$, denoted as feas $\left\{\operatorname{cone}\left(F_{k}\right)\right\}$. If cone $\left(F_{k}\right) \subseteq \mathcal{M}_{q}$ for certain feasible mode $q$ that $q \in$ feas $\left\{\operatorname{cone}\left(F_{k}\right)\right\}$, then label the cone cone $\left(F_{k}\right)$ as $\Omega^{q}$. An illustration of these concepts is given in Fig. 2.

A necessary and sufficient condition for switching stabilizability can now be presented, under the assumption that there is no sliding motion in the closed-loop switched system.

Theorem 1: Assume that there is no sliding motion in the closed-loop switched system. The switched linear system (2)-(3) with time-variant uncertainties can be globally asymptotically stabilized by a switching law (3), if and only if there exist matrices $L_{q}$, which satisfy the assumption (4) for each subsystem, such that the matrix

$$
\left[\begin{array}{c}
L_{1} \\
L_{2} \\
\vdots \\
L_{N}
\end{array}\right] \in \mathbb{R}^{\sum^{q} m_{q} \times n}
$$

has $n$ linear independent row vectors, and

$$
\bigcup_{q \in Q} \Omega^{q}=\mathbb{R}^{n} .
$$

\section{A. Sufficiency of Theorem 1}

This subsection is devoted to the sufficiency proof of Theorem 1. In the following, a stabilizing switching law is constructed and a global Lyapunov function is composed for the switched system. 
First, we give a necessary and sufficient condition for a polyhedron to be bounded. Note that a bounded polyhedron is called a polytope.

Lemma 3: [24] A nonempty polyhedral set, e.g.,

$$
\mathcal{P}=\left\{x \in \mathbb{R}^{n}: H x \leq g\right\}
$$

is bounded, if and only if the cone

$$
\mathcal{C}=\left\{X \in \mathbb{R}^{n}: H x \leq \overline{0}\right\}
$$

only contains the null vector $\overline{0}$.

For 0-symmetric polyhedrons, the boundedness checking can be reduced to the following simple rank condition.

Lemma 4: A nonempty 0-symmetric polyhedral set, e.g.,

$$
\mathcal{P}=\left\{x \in \mathbb{R}^{n}:|H x| \leq g\right\}
$$

is bounded, if and only if the matrix $H \in \mathbb{R}^{s \times n}(s \geq n$ ) has $n$ linear independent row vectors, or equivalently the rank of $H$ equals $n$.

In particular, for the intersection of $\mathcal{S}_{q}, \mathcal{S}$, we have the following corollary. The proof is given in the Appendix .

Corollary 1: If all the polyhedral sets $\mathcal{P}_{q}$ in $\mathbb{R}^{m_{q}}$ are 0 -symmetric, then so are the $\mathcal{S}_{q}$ in $\mathbb{R}^{n}$, for all $q \in Q=\{1,2, \ldots, N\}$. In addition, the intersection of all the polyhedral sets $\mathcal{S}_{q}$ is bounded, if and only if the matrix (13) has $n$ linear independent row vectors or, equivalently, it has rank $n$.

Notice that one may always make the level set of the polytopic Lyapunov function, $\mathcal{P}_{q}, 0$-symmetric [21]. From Corollary 1, we obtain that the rank condition (13) implies that the intersection of $\mathcal{S}_{q}$, namely the set $\mathcal{S}$, is a polyhedral C-set. Therefore, we may partition the state space $\mathbb{R}^{n}$ into finite cones which are induced by the facets of $\mathcal{S}$, and relabel these cones as $\Omega^{q}$. Because of condition (14), a conic partition of the whole state-space $\mathbb{R}^{n}$ is given by these $\Omega^{q}, q \in Q$. We define the switching law as

$$
x \in \Omega^{q} \Rightarrow \delta(x)=q .
$$

It can now be shown that this switching law can guarantee the uniformly ultimate boundedness (UUB) of the uncertain switched system (2)-(3) into $\mathcal{S}=\bigcap_{q \in Q} \mathcal{S}_{q}$; that is for any initial condition outside $\mathcal{S}$, there exists a finite time $T$ such that $x(t) \in \mathcal{S}$ for all $t \geq T$.

Proposition 1: Consider the class of switching laws defined by $\delta(x)=q$, when $x$ is contained in $\Omega^{q}$. Then, the uncertain continuous-time switched system (2)-(3) is UUB in the polyhedral C-set $\mathcal{S}=\bigcap_{q \in Q} \mathcal{S}_{q}$.

Proof: Consider the Minkowski function of $\mathcal{S}, V(x)$. For states outside $\mathcal{S}$, assume that $x(t) \in \Omega^{q}$ and current mode $\sigma(t)=q$. If no switching occurs at $t$, then there exists $\bar{\tau}>0$ such that $\forall 0<\tau \leq \bar{\tau}, x(t+\tau) \in \Omega^{q}$. So $V(x(t))=\Psi_{q}(x(t))$ and $V(x(t+\tau))=\Psi_{q}(x(t+\tau))$. Then, we obtain

$$
\mathcal{D}^{+} V(x(t))=\mathcal{D}^{+} \Psi_{q}(x(t))<0
$$

in view of Lemma 2 and the fact that $\Omega^{q} \subseteq \mathcal{M}_{q}$ by definition.

Else, if switching occurs at time $t$, then there exists $\bar{\tau}>0$ (due to no sliding motion assumption) such that $\forall 0<\tau \leq \bar{\tau}$, $x(t+\tau) \in \Omega_{q^{\prime}}$. Then $V(x(t))=\Psi_{q}(x(t))=\Psi_{q^{\prime}}(x(t))$ and
$V(x(t+\tau))=\Psi_{q^{\prime}}(x(t+\tau))$. The equality between $\Psi_{q}(x(t))$ and $\Psi_{q^{\prime}}(x(t))$ at the switching surface is simply because for all the states $x \in \Omega_{q} \cap \Omega_{q^{\prime}}$, which is the switching hyperplane passing the origin, $\Psi_{q}(x)=V(x)=\Psi_{q^{\prime}}(x)$ by definition. Here, $V(x)$ is the Minkowski function of $\mathcal{S}$ and continuous. Therefore

$$
\mathcal{D}^{+} V(x(t))=\mathcal{D}^{+} \Psi_{q^{\prime}}(x(t))<0
$$

for all states outside $\mathcal{S}$. Therefore, the uncertain switched system (2)-(3) is UUB with respect to the region $\bigcap_{q \in Q} \mathcal{S}_{q}$.

Because of Lemma 1 and the above UUB result, the switching control law can drive all the state trajectories into $\mu \mathcal{S}$ for all $\mu>$ 0 within a finite time interval. Select any decreasing sequence of $\mu_{k}$ with $\lim _{k \rightarrow \infty} \mu_{k}=0$; then all the trajectories will finally be driven to the origin. This implies globally asymptotic stability for the switched linear system (2)-(3).

This completes the sufficiency proof of Theorem 1.

\section{B. Necessity of Theorem 1}

To show the necessity of Theorem 1, we need the following lemma.

Lemma 5: Assume that no sliding motion occurs. A switched system can be globally asymptotically stabilized by a switching law if and only if it can be stabilized by a conic partition switching law.

Proof: Because of the fact that a conic partition switching law is a specific case of a switching law, the sufficiency is obvious.

To prove the necessity, it is assumed that the switched system can be globally asymptotically stabilized by a properly designed switching law for all initial conditions $x_{0} \in \mathbb{R}^{n}$. In particular, consider the unit sphere

$$
\Pi=\left\{x \in \mathbb{R}^{n}:\|x\|=1\right\}
$$

where $\|\cdot\|$ stands for the Euclidian norm of $\mathbb{R}^{n}$.

For any initial condition $x_{0} \in \Pi$, there exists a switching signal $\sigma(t)$ such that the trajectories starting from $x_{0}$ and following the dynamics:

$$
\dot{x}(t)=A_{\sigma(t)}(w) x(t)
$$

asymptotically converge to the origin.

In view of the results in [21] and [3] for uncertain linear timevariant systems, there exists a polyhedral C-set $\mathcal{P}_{\sigma}$ with $x_{0} \in$ $\partial \mathcal{P}_{\sigma}$, a positive constant $\bar{\tau}>0$ and a scalar $0<\lambda<1$, such that

$$
\left[I+\tau A_{\sigma(0)}(w)\right] x_{0} \subset \lambda \mathcal{P}_{\sigma}
$$

holds for all $0<\tau \leq \bar{\tau}$ and for all $w \in \mathcal{W}$. In addition, for any positive scalar $\mu>0$

$$
\left[I+\tau A_{\sigma(0)}(w)\right] \mu x_{0} \subset \lambda \mu \mathcal{P}_{\sigma}
$$

holds for all $0<\tau \leq \bar{\tau}$. Denote $\sigma(0) \in Q$ as $q_{0}$ in the sequel.

If we represent the polyhedral $\mathrm{C}$-set $\mathcal{P}_{\sigma}$ canonically as

$$
\mathcal{P}_{\sigma}(x)=\left\{x \in \mathbb{R}^{n}: f_{i} x \leq 1, i=1, \ldots, m\right\}
$$


then the Minkowski function $\Psi_{\mathcal{P}_{\sigma}}(x)$ can be written as

$$
\Psi_{\mathcal{P}_{\sigma}}(x)=\max _{1 \leq i \leq m}\left\{f_{i} x\right\}
$$

where $f_{i} \in \mathbb{R}^{1 \times n}$ is the gradient vector of facet $F_{i}$ of $\mathcal{P}_{\sigma}$, for $i=1, \ldots, m$.

Assume that for the initial condition $x_{0}, \Psi_{\mathcal{P}_{\sigma}}\left(x_{0}\right)=f_{i_{0}} x_{0}$. Then

$$
f_{i_{0}} x(\tau) \leq \Psi_{\mathcal{P}_{\sigma}}(x(\tau)) \leq \lambda \Psi_{\mathcal{P}_{\sigma}}\left(x_{0}\right)
$$

where $x(\tau)=\left[I+\tau A_{q_{0}}(w)\right] x_{0}$ for some $w \in \mathcal{W}$. This inequality holds uniformly for all $w \in \mathcal{W}$.

Therefore

$$
f_{i_{0}} x(\tau) \leq \lambda f_{i_{0}} x_{0} .
$$

Let us denote the hyperplane with which the facet $F_{i_{0}}$ is affiliated as $H_{x_{0}}$. The plane $H_{x_{0}}$ can be represented as

$$
H_{x_{0}}=\left\{x \in \mathbb{R}^{n}: f_{i_{0}}\left(x-x_{0}\right)=0\right\}
$$

or

$$
H_{x_{0}}=\left\{x \in \mathbb{R}^{n}: f_{i_{0}} x=1\right\} .
$$

We now define a scalar function $\psi_{H_{x_{0}}}(x)$ induced from the plane $H_{x_{0}}$ as

$$
\psi_{H_{x_{0}}}(x)=\inf \left\{\mu: x \in \mu H_{x_{0}}\right\}
$$

for all the points $x$ in $\mathbb{R}^{n}$.

Note that

$$
\Psi_{\mathcal{P}_{\sigma}}(x(0))=\psi_{H_{x_{0}}}\left(x_{0}\right) .
$$

Since for all $0<\tau<\bar{\tau}$,

$$
\psi_{H_{x_{0}}}\left(\left[I+\tau A_{q_{0}}(w)\right] x_{0}\right)-\psi_{H_{x_{0}}}\left(x_{0}\right)<0
$$

for all $w \in \mathcal{W}$, and for any positive scalar $\mu>0$, we have

$$
\psi_{H_{x_{0}}}\left(\left[I+\tau A_{q_{0}}(w)\right] \mu x_{0}\right)-\psi_{H_{x_{0}}}\left(\mu x_{0}\right)<0 .
$$

Similarly to the proof of Lemma 2, one can show that the Dini derivative of $\psi_{H_{x_{0}}}$ for all the states on the ray passing through $x_{0}$ is negative along the mode $q_{0}$.

Because of the continuity, there exists a small neighborhood of $x_{0}, B_{r}\left(x_{0}\right)$, such that for all $y \in \bigcap B_{r}\left(x_{0}\right)$, the Dini derivative of $\psi_{H_{x_{0}}}$ is negative. This procedure can be applied to all the points on the unit sphere $\Pi$, to obtain an open cover for $\Pi$ as

$$
\bigcup_{x \in \Pi} B_{x} \supseteq \Pi \text {. }
$$

Also based on the compactness of the unit sphere $\Pi$, we derive a finite cover, $B_{x_{i}}$ for $i=1, \ldots, M$

$$
\bigcup_{i=1}^{M} B_{x_{i}} \supseteq \Pi
$$

In addition, one may select these $x_{i}$ on the unit sphere $\Pi$ dense enough such that the intersection of a finite number of half-spaces

$$
\mathcal{P}=\bigcap_{i=1}^{M} H S_{x_{i}}
$$

where $H S_{x_{i}}=\left\{x \in \mathbb{R}^{n}: \psi_{H_{x_{i}}}(x) \leq 1\right\}$, is a bounded polyhedral set with the origin in its interior. Notice one may always choose the pair $x$ and $-x$ at the same time, so that the induced polyhedron $\mathcal{P}$ be 0 -symmetric.

Denote the Minkowski function of the polyhedral C-set $\mathcal{P}$, as $\Psi_{\mathcal{P}}(\cdot)$, expressed as

$$
\Psi_{\mathcal{P}}(x)=\max _{1 \leq i \leq M}\left\{\psi_{H_{x_{i}}}(x)\right\} .
$$

The function $\Psi_{\mathcal{P}}(x)$ is piecewise linear continuous function, and has the following properties.

1) Non-negativeness: $\Psi_{\mathcal{P}}(x) \geq 0, \Psi_{\mathcal{P}}(x)=0 \Leftrightarrow x=0$;

2) Radius-unboundedness: for any $\mu>0, \Psi_{\mathcal{P}}(\mu x)=$ $\mu \Psi_{\mathcal{P}}(x)$

3) Convexity: $\Psi_{\mathcal{P}}(x+y) \leq \Psi_{\mathcal{P}}(x)+\Psi_{\mathcal{P}}(y), \forall x, y \in \mathbb{R}^{n}$.

Basically, $\Psi_{\mathcal{P}}(x)$ defines a distance of $x$ from the origin which is linear in any direction.

Similarly to the conic partition switching law constructed in the sufficiency proof, we may induce from each facet of the polyhedral $\mathrm{C}$-set $\mathcal{P}$ a polyhedral convex cone, and assign a mode $q$ to guarantee the decreasing of $\Psi_{\mathcal{P}}(x)$. This generates a conic switching law which globally asymptotically stabilizes the switched system, and $\Psi_{\mathcal{P}}(x(t))$ serves as a (control) Lyapunov function for the switched system.

Because of Lemma 5, the existence of an asymptotically stabilizing switching law (without sliding motion) for the switched system (2)-(3) implies the existence of a conic partition based switching law which globally asymptotically stabilizes the closed-loop switched system. In the following, we will show that the existence of such conic partition based stabilizing switching law implies conditions (13) and (14).

Proof by Contradiction: Assume that there exists a point $\hat{x}$ such that $\hat{x}$ is not contained in the union of range spaces $\mathcal{M}_{q}$. Following the arguments in Lemma 5, the Dini derivative of $\Psi_{\mathcal{P}}(x)$ is negative along certain mode $q$ for all points $x=\mu \hat{x}$ with $\mu>0$. Since the level set of $\Psi_{\mathcal{P}}(x), \mathcal{P}$, is 0 -symmetric, the negativeness of the Dini derivative of $\Psi_{\mathcal{P}}(x)$ along mode $q$ holds for all $\mu \hat{x}$ with $\mu \neq 0$ as well. All the points $\mu \hat{x}$ form a linear subspace in $\mathbb{R}^{n}$ (a line passing through the origin), with $\mu=0$ corresponding to the origin. This linear subspace can be represented as a range space of a vector $R_{q}$, e.g., set $R_{q}=\hat{x}$. Notice that $\Psi_{\mathcal{P}}(x)$ is piecewise linear and can be represented as

$$
\Psi_{\mathcal{P}}(x)=\max _{i}\left\{\psi_{H_{x_{i}}}(x)\right\}=\max _{i}\left\{f_{i} x\right\} .
$$

Denote $i_{0}=\arg \max _{1 \leq i \leq M}\left\{f_{i} \hat{x}\right\}$ and select the row vector $f_{i_{0}}$ as $L_{q}$. Then, the negativeness of the Dini derivative of $\Psi_{\mathcal{P}}(x)$, i.e., $f_{i_{0}} A_{q}(w) \hat{x}<0$ for all $w \in \mathcal{W}$, implies that the auxiliary system

$$
\dot{\xi}(t)=L_{q} A_{q}(w) R_{q} \xi(t)
$$


which is a scalar system with negative right hand side, is asymptotically stable. The condition that $L_{q} R_{q}=1$ can be easily satisfied by a scaling factor since $L_{q} R_{q}=f_{i_{0}} \hat{x}>0$. This implies that the point $\hat{x}$ is contained in $\mathcal{M}_{q}$, which leads to a contradiction.

Next, we show that the rank condition (13) is also necessary for the construction of a conic partition switching law. Let us denote the conic partition derived in the proof of Lemma 5 as $\Omega^{q}$, then

$$
\bigcup_{q \in Q} \Omega^{q}=\mathbb{R}^{n}
$$

As proved in Lemma 5, within the cone $\Omega^{q}$, the Dini derivative of $\Psi_{\mathcal{P}}(x)$ is negative along the dynamics of the mode $q$. This means that the cone

$$
\mathcal{C}=\bigcap_{q} \mathcal{C}_{q}
$$

contains only the null vector, which implies that the intersection of $\mathcal{S}_{q}(q \in Q)$ is bounded. By Corollary 1, the rank condition (13) is obtained.

This completes the necessity proof.

\section{HANDLING SLIDING Motion}

So far, all our arguments are under the assumption that no sliding motion is generated by the switched systems. However, sliding motions may occur through the proposed conic partition based switching laws. It is also possible that the generated sliding motion causes instability in the closed-loop switched systems. Therefore, it is important to explicitly consider sliding motions. Similar issues arise in the methods for switching stabilization based on piecewise quadratic Lyapunov functions, where special care needs to be taken; see, e.g., [26]. In this section, we identify conditions under which unstable sliding motions can be avoided. We further show that these conditions are automatically satisfied in the cases of stable sliding motion and stable nonsliding switchings. Finally, a more general statement of the stabilizability result is presented.

We first consider a way to distinguish a stable sliding motion from an unstable one.

Without loss of generality, assume that a sliding motion occurs between the subsystem $q_{1}$ and $q_{2}$ along the intersection hyperplane of conic regions $\Omega^{q_{1}}$ and $\Omega^{q_{2}}$. From the sufficiency proof in Section IV-A, we know that these conic regions $\Omega^{q_{1}}$ and $\Omega^{q_{2}}$ are cones generated from two facets, e.g., $F_{1}$ and $F_{2}$, respectively. Let the hyperplane that corresponds to the facet $F_{k}$ be denoted as

$$
\mathrm{H}_{k}=\left\{x \in \mathbb{R}^{n}: f_{k} x=1\right\}
$$

where $f_{k} \in \mathbb{R}^{1 \times n}$ is the corresponding gradient vector of facet $F_{k}(k=1,2$ here). Therefore, the sliding motion is contained in the hyperplane $\left(f_{1}-f_{2}\right) x=0$, which is passing through the origin.

If the sliding motion is stable, then one may pick appropriate $L_{q_{1} q_{2}}$ and $R_{q_{1} q_{2}}$ to project the trajectories of closed-loop switched systems to the sliding hyperplane $\left(f_{1}-f_{2}\right) x=0$.

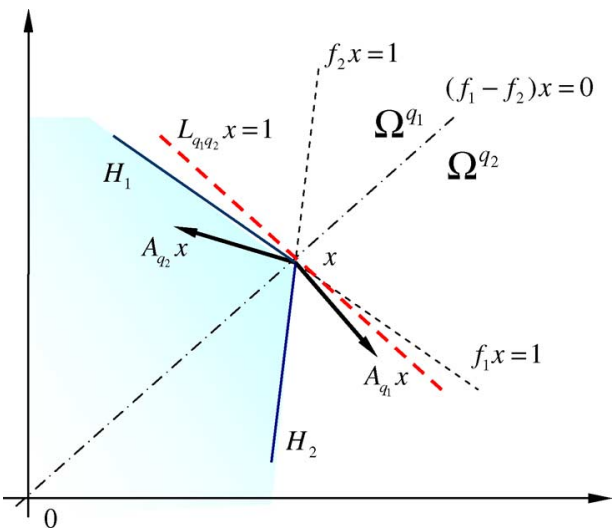

Fig. 3. Case of stable sliding motion.

Because the sliding motion is stable, the projected one-dimensional dynamics are stable as well, that is

$$
L_{q_{1} q_{2}}\left[\theta A_{q_{1}}(w)+(1-\theta) A_{q_{2}}(w)\right] R_{q_{1} q_{2}}<0
$$

for $\theta \in[0,1]$ and $w \in \mathcal{W}$.

For example, one may set $L_{q_{1} q_{2}}$ as $x^{T} /\|x\|^{2}$ and $R_{q_{1} q_{2}}=x$, where $x$ is an nonzero point on the sliding hyperplane, $\left(f_{1}-\right.$ $\left.f_{2}\right) x=0$. Another interpretation of the previous inequality is that all the vectors $A_{q_{1}}(w) x$ and $A_{q_{2}}(w) x$ point to the negative side of the plane $L_{q_{1} q_{2}} x=1$ for all $w \in \mathcal{W}$ and $x$ on the sliding surface. The case of stable sling motion is illustrated in Fig. 3.

Therefore, for the case of stable sliding motion, one can always find a row vector $L_{q_{1} q_{2}}$ such that

$$
L_{q_{1} q_{2}}\left[\theta A_{q_{1}}(w)+(1-\theta) A_{q_{2}}(w)\right] R_{q_{1} q_{2}}<0
$$

for all $\theta \in[0,1]$ and $w \in \mathcal{W}$. In addition, $L_{q_{1} q_{2}} R_{q_{1} q_{2}} \neq 0$.

If now the sliding motion is unstable, the trajectories will go to infinity along the switching hyperplane.

This implies that the sliding motion generates $\dot{x}=\lambda I_{n} x$ kind of dynamics. Therefore, in view of earlier discussion, one cannot find $L_{q_{1} q_{2}}$ and $R_{q_{1} q_{2}}\left(L_{q_{1} q_{2}} R_{q_{1} q_{2}} \neq 0\right)$ such that

$$
L_{q_{1} q_{2}}\left[\theta A_{q_{1}}(w)+(1-\theta) A_{q_{2}}(w)\right] R_{q_{1} q_{2}}<0
$$

for $\theta \in[0,1]$ and $w \in \mathcal{W}$. In fact, for certain values of $\theta \in[0,1]$ and $w \in \mathcal{W}$, the matrix $\theta A_{q_{1}}(w)+(1-\theta) A_{q_{2}}(w)$ is similar to $\lambda I_{n}$ for some positive real $\lambda$. This is illustrated in Fig. 4 .

So the existence of such $L_{q_{1} q_{2}}$ can serve as a distinctive feature between stable and unstable sliding motions. It is possible to include such requirement in the design procedure so to avoid unstable sliding motions. The next question is whether this additional requirement will cause the loss of necessity. Fortunately, this condition is automatically satisfied for the case of stable nonsliding switchings as well.

To see this, for the states $x$ on the switching surface, $\left(f_{1}-\right.$ $\left.f_{2}\right) x=0$, if no sliding motion occur, then both $A_{q_{1}}(w) x$ and $A_{q_{2}}(w) x$ point to the same side of the switching hyperplane, for example the positive side as illustrated in Fig. 5.

In addition, if the switched system is stabilizable, i.e, satisfying the conditions in Theorem 1, then $A_{q_{1}}(w) x$ points towards the negative side of $f_{1} x=1$ and $A_{q_{2}}(w) x$ points towards the negative side of $f_{2} x=1$. 


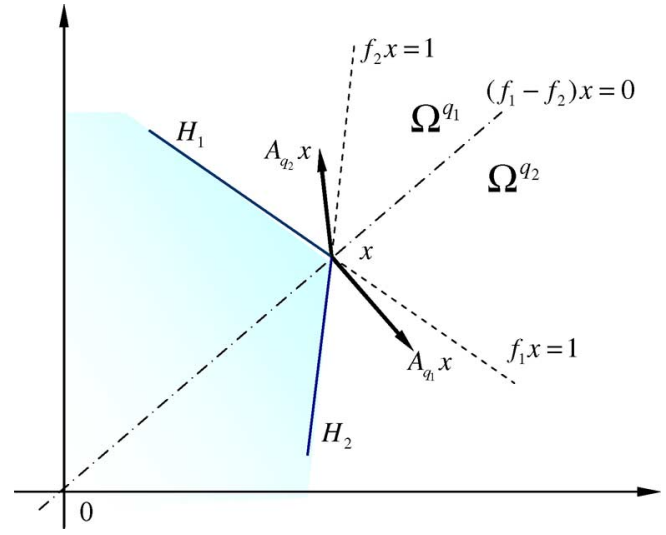

Fig. 4. Case of unstable sliding motion.

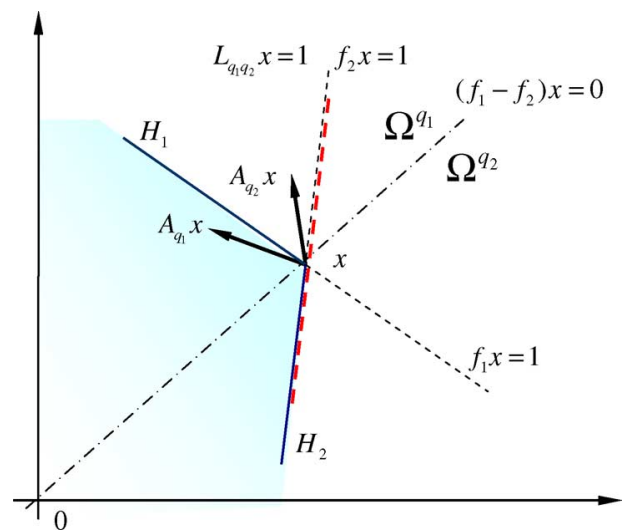

Fig. 5. Case of no sliding motion occurring.

For the case illustrated in Fig. 5, the vector $L_{q_{1} q_{2}}$ may be selected as $f_{2}$, while $R_{q_{1} q_{2}}=x$ for $x$ on the the hyperplane $\left(f_{1}-f_{2}\right) x=0$.

By construction, $A_{q_{1}}(w) x$ points towards the negative side of $L_{q_{1} q_{2}} x=1$. Actually, $A_{q_{2}}(w) x$ also points towards the negative side of $L_{q_{1} q_{2}} x=1$. This is simply because of convexity. Note that the intersection of the negative side of $f_{1} x=1$ and the positive side of $\left(f_{1}-f_{2}\right) x=0$ is contained inside the negative side of $L_{q_{1} q_{2}} x=1$. Therefore, both

$$
\text { and } \begin{aligned}
L_{q_{1} q_{2}} A_{q_{2}}(w) R_{q_{1} q_{2}} & <0 \\
L_{q_{1} q_{2}} A_{q_{1}}(w) R_{q_{1} q_{2}} & <0
\end{aligned}
$$

hold for all $w \in \mathcal{W}$. Hence

$$
L_{q_{1} q_{2}}\left[\theta A_{q_{1}}(w)+(1-\theta) A_{q_{2}}(w)\right] R_{q_{1} q_{2}}<0
$$

for all $\theta \in[0,1]$ and $w \in \mathcal{W}$. The argument is the same for the case when both $A_{q_{1}}(w) x$ and $A_{q_{2}}(w) x$ point to the negative side of the switching hyperplane.

In view of this, the additional requirement of common $L$ and $R$ on the switching surface is not conservative, in the sense that it excludes exactly the unstable sliding motions; and, clearly, a switched system is stabilizable only when it can be done so without unstable sliding motions. Therefore, the proposed necessary and sufficient condition for the asymptotic switching stabilizability can be extended as follows.

Theorem 2: The switched linear system (2)-(3) with timevariant uncertainties can be globally asymptotically stabilized by a (static state feedback) switching law (3), if and only if

1) there exist matrices $L_{q}$, which satisfy the assumption (4) for each subsystem and the rank condition (13);

2) the induced conic cones cover the whole state space, i.e.,

$$
\bigcup_{q \in Q} \Omega^{q}=\mathbb{R}^{n}
$$

3) when $\Omega^{q_{i}} \cap \Omega^{q_{j}} \neq \emptyset$, there exists a row vector $L_{q_{i} q_{j}}$ such that

$$
L_{q_{i} q_{j}}\left[\theta A_{q_{i}}(w)+(1-\theta) A_{q_{j}}(w)\right] R_{q_{i} q_{j}}<0
$$

for $\theta \in[0,1]$ and $w \in \mathcal{W}$. Here, $R_{q_{i} q_{j}}$ is selected such that $L_{q_{i} q_{j}} R_{q_{i} q_{j}} \neq 0$ and $\Omega^{q_{i}} \cap \Omega^{q_{j}}$ is contained in the range space of $R_{q_{i} q_{j}}$.

Note that the first two conditions are exactly the same as Theorem 1 , while condition (3) is added to exclude possible unstable sliding motions.

\section{NUMERICAL EXAMPLE}

To illustrate the results, let us consider the following example. A similar example without parametric uncertainties was used in [8] to show that switching between two unstable systems may exhibit stable behavior.

Example 3: Consider the following continuous-time switched linear system:

$$
\dot{x}(t)= \begin{cases}A_{1}(w) x(t), & \sigma(t)=q_{1} \\ A_{2}(w) x(t), & \sigma(t)=q_{2} .\end{cases}
$$

In this example, the mode set $Q=\left\{q_{1}, q_{2}\right\}$, and the corresponding state matrices for each subsystem are given by

$$
\begin{aligned}
& A_{1}(w)=\left[\begin{array}{cc}
0 & 10+w \\
0 & 0
\end{array}\right] \\
& A_{2}(w)=\left[\begin{array}{cc}
1.5+w & 2 \\
-2 & -0.5+w
\end{array}\right]
\end{aligned}
$$

where $w \in[-1,0]$.

For the first subsystem, the existence of stable auxiliary systems and the parameterization of the corresponding $L_{1}$ and $R_{1}$ have been studied in Example 2. For the second subsystem, if we let $L_{2}=\left[\begin{array}{ll}1 & 1\end{array}\right]$ and parameterize $R_{2}$ as $\left[\begin{array}{c}r \\ 1-r\end{array}\right]$, then

$$
\begin{aligned}
L_{2} A_{2} R_{2} & =\left[\begin{array}{ll}
1 & 1
\end{array}\right]\left[\begin{array}{cc}
1.5+w & 2 \\
-2 & -0.5+w
\end{array}\right]\left[\begin{array}{c}
r \\
1-r
\end{array}\right] \\
& =1.5-2 r+w .
\end{aligned}
$$

The auxiliary system for mode $q_{2}$ can be written as

$$
\dot{\xi}(t)=(1.5-2 r+w) \xi(t)
$$


which is asymptotically stable for $r>0.75$. The interval $[-1,1]$ is contractive for the second auxiliary system, which induces the polyhedral region

$$
\mathcal{S}_{2}=\left\{x \in \mathbb{R}^{2} \mid-1 \leq\left[\begin{array}{ll}
1 & 1
\end{array}\right] x \leq 1\right\}
$$

The range space $\mathcal{M}_{2}$ can be determined as the sector region between line $x_{2}=1 / 3 x_{1}$ and line $x_{2}=-x_{1}$, and it is shown in Fig. 6.

It is clear from the sufficiency proof that the active region, i.e., $\Omega^{q}$ of each mode, should be constrained inside $\mathcal{M}_{q}$. Therefore, we select a switching surface candidate inside $\mathcal{M}_{2}$ as the line $x_{2}=0.3 x_{1}$, which intersect the boundary of $\mathcal{S}_{2}$ at point $p_{1}=$ $(10 / 13,3 / 13)$ and $p_{3}=(-10 / 13,-3 / 13)$.

For the first subsystem, if we select $L_{1}=\left[\begin{array}{ll}-1 & 20\end{array}\right]$ and $R_{1}=\left[\begin{array}{c}20 r-1 \\ r\end{array}\right]$, then

$$
\begin{aligned}
L_{1} A_{1} R_{1} & =\left[\begin{array}{ll}
-1 & 20
\end{array}\right]\left[\begin{array}{cc}
0 & 10+w \\
0 & 0
\end{array}\right]\left[\begin{array}{c}
20 r-1 \\
r
\end{array}\right] \\
& =-(10+w) r .
\end{aligned}
$$

The auxiliary system for mode $q_{1}$ can be written as

$$
\dot{\xi}(t)=-(10+w) r \xi(t)
$$

which is asymptotically stable when $r>0$.

If one selects $\mathcal{P}_{1}$ as the interval $[-3.85,3.85]$, which is contractive for the above auxiliary system, then the polyhedral region

$$
\mathcal{S}_{1}=\left\{x \in \mathbb{R}^{2} \mid-3.85 \leq\left[\begin{array}{ll}
-1 & 20
\end{array}\right] x \leq 3.85\right\}
$$

is obtained. $\mathcal{S}_{1}$ and $\mathcal{M}_{1}$ are shown in Fig. 6.

For this choice, the points $p_{1}$ and $p_{3}$ are among the intersection points of the boundaries of $\mathcal{S}_{1}$ and $\mathcal{S}_{2}$. Another intersection point for the boundaries of $\mathcal{S}_{1}$ and $\mathcal{S}_{2}$ are $p_{2}=(-1.135,0.135)$ and $p_{4}=(1.135,-0.135)$. Connecting the origin and $p_{2}$, we obtain the other switching surface candidate $x_{2}=-0.119 x_{1}$, which also passes $p_{4}=(1.135,-0.135)$.

Note that the rank condition (13) is satisfied, namely

$$
\operatorname{rank}\left[\begin{array}{c}
L_{1} \\
L_{2}
\end{array}\right]=\operatorname{rank}\left[\begin{array}{cc}
-1 & 20 \\
1 & 1
\end{array}\right]=2 .
$$

Hence, the intersection of $\mathcal{S}_{1}$ and $\mathcal{S}_{2}$ is a polyhedral C-set, which has $p_{1}, p_{2}, p_{3}$, and $p_{4}$ as its vertices. In particular

$$
\begin{aligned}
\mathcal{S} & =\mathcal{S}_{1} \bigcap \mathcal{S}_{2} \\
& =\left\{x \in \mathbb{R}^{2} \mid\left[\begin{array}{c}
-3.85 \\
-1
\end{array}\right] \leq\left[\begin{array}{cc}
-1 & 20 \\
1 & 1
\end{array}\right] x \leq\left[\begin{array}{c}
3.85 \\
1
\end{array}\right]\right\}
\end{aligned}
$$

which is 0 -symmetric. The (control) polytopic Lyapunov function induced from $\mathcal{S}$ can be represented as

$$
V(x)=\max \left\{\frac{|[-1,20] x|}{3.85},|[1,1] x|\right\} .
$$
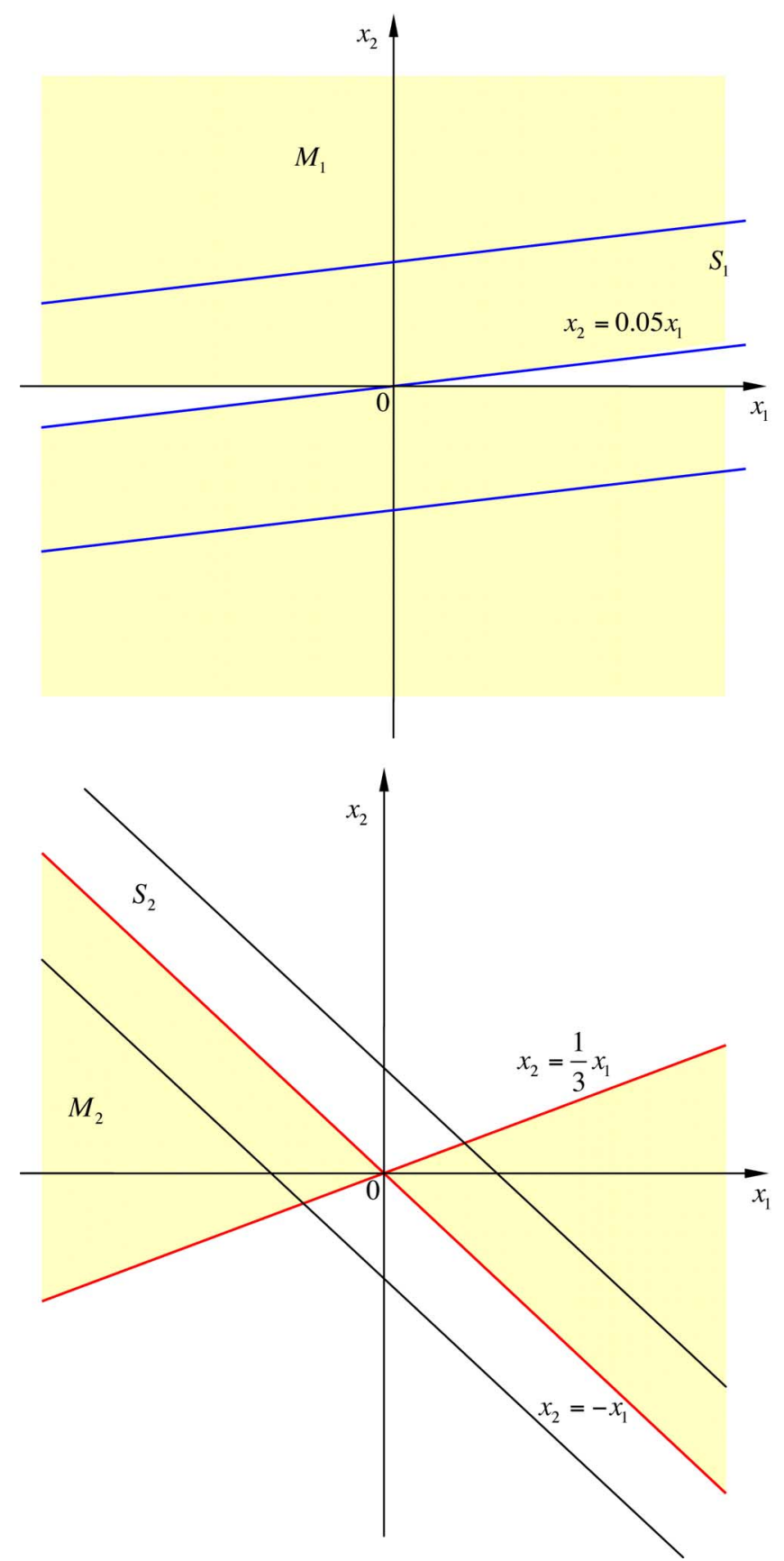

Fig. 6. Illustration of $\mathcal{S}_{1}, \mathcal{M}_{1}, \mathcal{S}_{2}$, and $\mathcal{M}_{2}$.

The state-space $\mathbb{R}^{2}$ is divided into four cones corresponding to the four facets of $\mathcal{S}$. For example, the cone generated by the facet between vertices $p_{1}$ and $p_{2}$, denoted as cone $\left(p_{1}, p_{2}\right)$ with some abuse of notation, has $q_{1}$ as its active mode and cone $\left(p_{1}, p_{2}\right) \subset \mathcal{M}_{1}$. Relabel cone $\left(p_{1}, p_{2}\right)$ as $\Omega^{1}$. Similarly, relabel the other three cones and the condition (14) is verified. Therefore, the switched linear systems can be globally asymptotically stabilized through proper switching, and the stabilizing switching control law can be constructed following (15).

To verify that the Dini derivative of $V(x)$ is negative following the conic partition switching law, pick a state $x_{0}=$ $[6,1]^{T}$, which lies in $\Omega^{2}$. Note that

$$
V\left(x_{0}\right)=\max \left\{\frac{\left|[-1,20] x_{0}\right|}{3.85},\left|[1,1] x_{0}\right|\right\}=[1,1] x_{0}=7 \text {. }
$$


So, following the second mode:

$$
\begin{aligned}
\mathcal{D}^{+} V\left(x_{0}\right) & =\max _{w}\left[\begin{array}{ll}
1 & 1
\end{array}\right] A_{2}(w) x_{0} \\
& =\max _{w}\left[\begin{array}{ll}
1 & 1
\end{array}\right]\left[\begin{array}{cc}
1.5+w & 2 \\
-2 & -0.5+w
\end{array}\right]\left[\begin{array}{l}
6 \\
1
\end{array}\right] \\
& =\max _{w}(-1.5+7 w)=-1.5<0
\end{aligned}
$$

where $w \in[-1,0]$.

To make sure that no unstable sliding motion occurs, we need to check condition (3) in Theorem 2. For illustration, consider the switching surface $x_{2}=0.3 x_{1}$, select $L_{q_{1} q_{2}}=L_{1}=\left[\begin{array}{ll}-1 & 20\end{array}\right]$, and $R_{q_{1} q_{2}}=\left[\begin{array}{ll}1 & 0.3\end{array}\right]^{T}$, then

$$
\begin{aligned}
L_{q_{1} q_{2}} A_{1}(w) R_{q_{1} q_{2}} & =\left[\begin{array}{ll}
-1 & 20
\end{array}\right]\left[\begin{array}{cc}
0 & 10+w \\
0 & 0
\end{array}\right]\left[\begin{array}{c}
1 \\
0.3
\end{array}\right] \\
& =-(3+0.3 w)<0
\end{aligned}
$$

for all $w \in[-1,0]$, and

$$
\begin{aligned}
L_{q_{1} q_{2}} A_{2}(w) R_{q_{1} q_{2}} & =\left[\begin{array}{ll}
-1 & 20
\end{array}\right]\left[\begin{array}{cc}
1.5+w & 2 \\
-2 & -0.5+w
\end{array}\right]\left[\begin{array}{c}
1 \\
0.3
\end{array}\right] \\
& =-45.1+5 w<0
\end{aligned}
$$

for all $w \in[-1,0]$ as well. Similarly, it can be checked for the switching surface $x_{2}=-0.119 x_{1}$. Therefore, the designed switching law stabilizes the switched system.

This gives us a switching control law quite similar to the one proposed in [8]; see [8] for simulations. Using our approach, it is possible to derive different stabilizing switching laws for this example by choosing different $L_{q}$ and/or different contractive regions for the auxiliary systems. In fact, one may obtain a whole class of switching control laws that asymptotically stabilize this switched system.

\section{CONCLUDING REMARKS}

In this paper, continuous-time switched linear systems affected by parameter variations were considered, and the switching stabilizability problem for such uncertain switched linear systems was investigated. A necessary and sufficient condition for the existence of a switching control law (in a static state feedback form) to assure the asymptotic stability of the closed-loop switched systems was derived. It is also very interesting to note that if a switched linear system can be asymptotically stabilized by a static state feedback switching law without sliding motion, then one can always implement it in a conic partition based switching law. However, it is not known yet whether a switching stabilizable switched system can always be stabilized by a switching law in a static state feedback form.

Although the conditions given in this paper were proved to be necessary and sufficient, the checking of the necessity is not easy, as it requires to parameterize all $L_{q}$ and $R_{q}$ that satisfy (4). The calculation of such $L_{q}$ and $R_{q}$ for a given subsystem could be tedious, and systematic approaches need to be developed for parameterization of such generalized similarity matrices. Fortunately, it is always possible to restrict the search to the vector case, i.e., $m_{q}=1, L_{q} \in \mathbb{R}^{1 \times n}$, and $R_{q} \in \mathbb{R}^{n \times 1}$. This makes it is possible to formulate the determination of $L_{q}$ and $R_{q}$ into an optimization problem. In addition, for the parametric uncertainty case, it is possible to represent the uncertain dynamics as an equivalent poly-

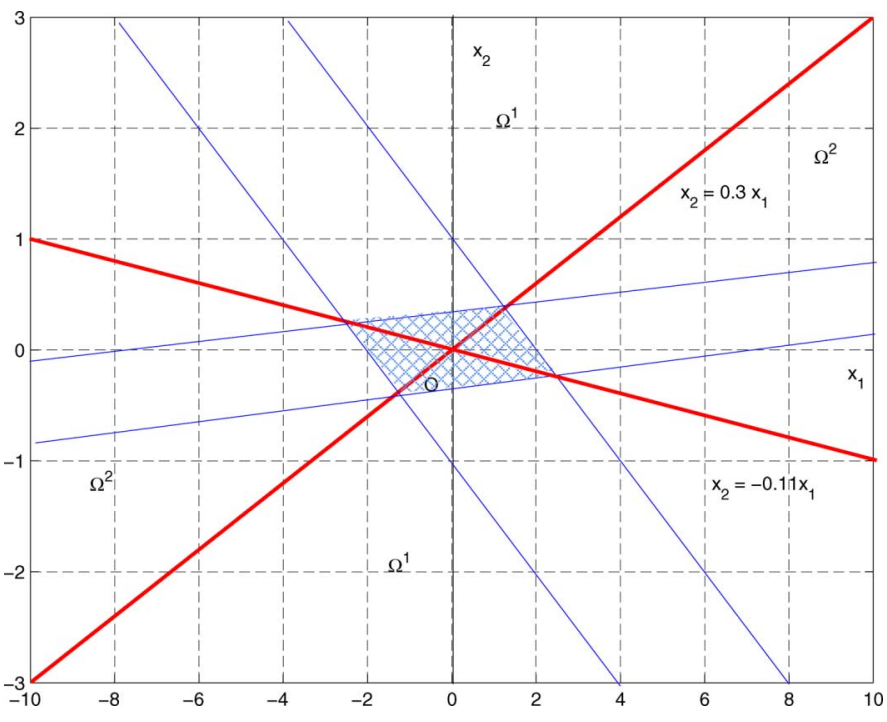

Fig. 7. Intersection of $\mathcal{S}_{1}$ and $\mathcal{S}_{2}$ and its induced conic partition-based stabilizing switching law.

topic uncertain different inclusion. The benefit of formulating it as a polytopic uncertainty is that in calculation one only needs to consider the finite vertex matrices. Nevertheless, the properties of such generalized similarity transformations and the parameterization of such $L_{q}$ and $R_{q}$ need further study.

\section{APPENDIX \\ PROOF OF COROLlaRY 1}

Assume that the 0 -symmetric polyhedral set $\mathcal{P}_{q}$ has the following form:

$$
\mathcal{P}_{q}=\left\{\xi \in \mathbb{R}^{m_{q}}:\left|F_{q} \xi\right| \leq \overline{1}\right\} .
$$

Then

$$
\mathcal{S}_{q}=\left\{x \in \mathbb{R}^{n}:-\overline{1} \leq F_{q} L_{q} x \leq \overline{1}\right\} .
$$

Hence, $\mathcal{S}_{q}$ is 0 -symmetric, so is the intersection of $\mathcal{S}_{q}$

$$
\mathcal{S}=\bigcap_{q \in Q} \mathcal{S}_{q}=\left\{x \in \mathbb{R}^{n}: \overline{1} \leq\left[\begin{array}{c}
F_{1} L_{1} \\
F_{2} L_{2} \\
\vdots \\
F_{N} L_{N}
\end{array}\right] x \leq \overline{1}\right\} .
$$

The nonemptiness is easy to verify by observing that the origin is contained in $\mathcal{S}_{q}$ for all $q \in Q$.

By Lemma $4, \mathcal{S}$ is bounded if and only if the matrix

$$
\left[\begin{array}{c}
F_{1} L_{1} \\
F_{2} L_{2} \\
\vdots \\
F_{N} L_{N}
\end{array}\right] \in \mathbb{R}^{\sum s_{q} \times n}
$$

has $n$ linear independent row vectors, or namely its rank equals $n$.

Note that $F_{q} \in R^{s_{q} \times m_{q}}, s_{q} \geq m_{q}$, and $F_{q}$ has rank $m_{q}$, where $m_{q} \leq n$

$$
\left[\begin{array}{c}
F_{1} L_{1} \\
F_{2} L_{2} \\
\vdots \\
F_{N} L_{N}
\end{array}\right]=\left[\begin{array}{cccc}
F_{1} & 0 & \cdots & 0 \\
0 & F_{2} & \cdots & 0 \\
\vdots & \vdots & \ddots & \vdots \\
0 & \cdots & \cdots & F_{N}
\end{array}\right]\left[\begin{array}{c}
L_{1} \\
L_{2} \\
\vdots \\
L_{N}
\end{array}\right] .
$$


Because each $F_{q}$ has rank $m_{q}$, the rank of $\operatorname{diag}\left\{F_{q}\right\}$ equals $\sum_{q \in Q} m_{q}$, which is greater or equal to $n$.

Therefore, because of the Sylvester rank inequality, the rank of the matrix (13) equals $n$.

\section{ACKNOWLEDGMENT}

The authors would like to thank Dr. A. Bemporad, Dr. R. DeCarlo, Dr. M. Egerstedt, Dr. S. Ge, Dr. J. Hespanha, Dr. D. Liberzon, Dr. A. P. Molchanov, Dr. S. Pettersson, Dr. P. Tabuada, Dr. X. Xu, and the anonymous reviewers for their valuable comments.

\section{REFERENCES}

[1] A. Bemporad, G. Ferrari-Trecate, and M. Morari, "Observability and controllability of piecewise affine and hybrid systems," IEEE Trans. Autom. Control, vol. 45, no. 10, pp. 1864-1876, Oct. 2000.

[2] A. Bemporad and M. Morari, "Control of systems integrating logic, dynamics, and constraints," Automatica, vol. 35, no. 3, pp. 407-427, 1999.

[3] F. Blanchini, "Nonquadratic Lyapunov functions for robust control," Automatica, vol. 31, no. 3, pp. 451-461, 1995.

[4] D. Cheng, L. Guo, Y. Lin, and Y. Wang, "Stabilization of switched linear systems," IEEE Trans. Autom. Control, vol. 50, no. 5, pp. 661-666, May 2005.

[5] D. Corona, A. Giua, and C. Seatzu, "Stabilization of switched systems via optimal control," in Proc. 16th IFAC World Congr., 2005.

[6] F. A. Cuzzola and M. Morari, "An LMI approach for $\mathcal{H}_{\infty}$ analysis and control of discrete-time piecewise affine systems," Int. J. Control, vol. 75, no. 16-17, pp. 1293-1301, 2002.

[7] J. Daafouz, R. Riedinger, and C. Iung, "Stability analysis and control synthesis for switched systems: A switched Lyapunov function approach," IEEE Trans. Autom. Control, vol. 47, no. 11, pp. 1883-1887, Nov. 2002.

[8] R. A. Decarlo, M. S. Branicky, S. Pettersson, and B. Lennartson, "Perspectives and results on the stability and stabilizability of hybrid systems," in Proceedings of the IEEE: Special Issue on Hybrid Systems, P. J. Antsaklis, Ed. New York: IEEE Press, 2000, vol. 88, pp. 1069-1082.

[9] K. Drayton and C. Tong, "Constructive stability and asymptotic stability of dynamical systems," IEEE Trans. Circuits Syst., vol. CAS-27, no. 11, pp. 1121-1130, Nov. 1980.

[10] M. Egerstedt and M. Babaali, "On observability and reachability in a class of discrete-time switched linear systems," in Proc. 2005 Amer. Control Conf., 2005, pp. 1179-1180.

[11] L. Fang, H. Lin, and P. J. Antsaklis, "Stabilization and performance analysis for a class of switched systems," in Proc. 43rd IEEE Conf. Decision Control, 2004, pp. 3265-3270.

[12] E. Feron, Quadratic stabilizability of switched systems via state and output feedback MIT, Cambridge, MA, 1996, Tech. Rep. CICS-P-468.

[13] A. Hassibi, S. P. Boyd, and J. P. How, "Control of asynchronous dynamical systems with rate constraints on events," in Proc. 38th IEEE Conf. Decision Control, 1999, pp. 1345-1351.

[14] J. P. Hespanha, D. Liberzon, D. Angeli, and E. D. Sontag, "Nonlinear norm-observability notions and stability of switched systems," IEEE Trans. Autom. Control, vol. 52, no. 1, pp. 154-168, Jan. 2005.

[15] H. Ishii, T. Basar, and R. Tempo, "Randomized algorithms for synthesis of switching rules for multimodal systems," IEEE Trans. Autom. Control, vol. 50, no. 6, pp. 754-767, Jun. 2005.

[16] M. Johansson, "Piecewise Linear Control Systems," Ph.D. dissertation, Lund Institute of Technology, Lund, Sweden, 1999.

[17] M. Lazar, M. Heemels, S. Weiland, A. Bemporad, and O. Pastravanu, "Infinity norms as Lyapunov functions for model predictive control of constrained PWA systems," in Hybrid Systems: Computation and Control. New York: Springer-Verlag, 2005, vol. 3414, Lecture Notes in Computer Science, pp. 417-432.

[18] D. Liberzon, Switching in Systems and Control. Boston, MA: Birkhäuser, 2003.

[19] D. Liberzon and A. S. Morse, "Basic problems in stability and design of switched systems," IEEE Control Syst. Mag., vol. 19, no. 5, pp. 59-70, Oct. 1999.
[20] H. Lin and P. J. Antsaklis, "Stability and stabilizability of switched linear systems: A short survey of recent results," in Proc. ISIC-MED Joint Conf., 2005, pp. 24-29.

[21] D. Liu and A. Molchanov, "Criteria for robust absolute stability of time-varying nonlinear continuous-time systems," Automatica, vol. 38 , no. 4, pp. 627-637, 2002.

[22] A. N. Michel, "Recent trends in the stability analysis of hybrid dynamical systems," IEEE Trans. Circuits Syst. I, vol. 46, no. 1, pp. 120-134, Jan. 1999.

[23] A. P. Molchanov and E. Pyatnitskiy, "Criteria of asymptotic stability of differential and difference inclusions encountered in control theory," Syst. Control Lett., vol. 13, pp. 59-64, 1989.

[24] M. J. Panik, Fundamentals of Convex Analysis. Norwell, MA: Kluwer, 1993.

[25] S. Pettersson, "Synthesis of switched linear systems," in Proc. 42nd IEEE Conf. Decision Control, 2003, pp. 5283-5288.

[26] _ - "Synthesis of switched linear systems handling sliding motions," in Proc. Int. Symp. Intelligent Control, 2005, pp. 18-23.

[27] S. Pettersson and B. Lennartson, "Stabilization of hybrid systems using a min-projection strategy," in Proc. Amer. Control Conf., 2001, pp. 223-228.

[28] A. Polański, "On absolute stability analysis by polyhedral Lyapunov functions," Automatica, vol. 36, no. 4, pp. 573-578, 2000.

[29] L. Rodrigues and S. Boyd, "Piecewise-affine state feedback for piecewise-affine slab systems using convex optimization," Syst. Control Lett., vol. 54, no. 9, pp. 835-853, 2005.

[30] L. Rodrigues, A. Hassibi, and J. P. How, "Observer-based control of piecewise-affine systems," Int. J. Control, vol. 76, no. 5, pp. 459-477, 2003.

[31] E. Skafidas, R. J. Evans, A. V. Savkin, and I. R. Petersen, "Stability results for switched controller systems," Automatica, vol. 35, no. 4, pp. 553-564, 1999

[32] Z. Sun, "Stabilizability and insensitivity of switched linear systems," IEEE Trans. Autom. Control, vol. 49, no. 7, pp. 1133-1137, Jul. 2004

[33] Z. Sun and S. S. Ge, "Analysis and synthesis of switched linear control systems," Automatica, vol. 41, no. 2, pp. 181-195, 2005.

[34] — Switched Linear Systems: Control and Design. New York: Springer-Verlag, 2005

[35] Z. Sun, S. S. Ge, and T. H. Lee, "Controllability and reachability criteria for switched linear systems," Automatica, vol. 38, no. 5, pp. 775-786, 2002.

[36] M. A. Wicks and R. A. DeCarlo, "Solution of coupled Lyapunov equations for the stabilization of multi-modal linear systems," in Proc. Amer. Control Conf., 1997, pp. 1709-1713.

[37] M. A. Wicks, P. Peleties, and R. A. DeCarlo, "Switched controller design for the quadratic stabilization of a pair of unstable linear systems," Eur. J. Control, vol. 4, pp. 140-147, 1998

[38] X. Xu and P. J. Antsaklis, "Stabilization of second-order LTI switched systems," Int. J. Control, vol. 73, no. 14, pp. 1261-1279, 2000.

[39] —_, "Optimal control of switched systems based on parameterization of the switching instants," IEEE Trans. Autom. Control, vol. 49, no. 1, pp. 2-16, Jan. 2004.

[40] C. Yfoulis and R. Shorten, "A numerical technique for stability analysis of linear switched systems," Int. J. Control, vol. 77, no. 11, pp. 1019-1039, Jul. 2004.

[41] G. Zhai, H. Lin, and P. J. Antsaklis, "Quadratic stabilizability of switched linear systems with polytopic uncertainties," Int. J. Control, vol. 76, no. 7, pp. 747-753, 2003.

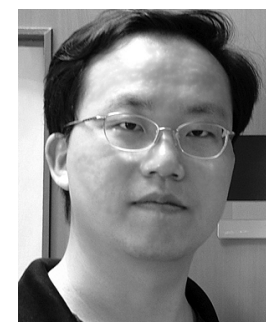

Hai Lin (M'04) received the B.S. degree from the University of Science and Technology, Beijing, China, in 1997, the M.Eng. degree from the Chinese Academy of Science, China, in 2000, and the Ph.D. degree from the University of Notre Dame, Notre Dame, IN, in 2005

He is currently an Assistant Professor in the Electrica and Computer Engineering Department at the National University of Singapore. His research interests are in the multidisciplinary study of the problems at the intersection of control, communication, computation, and life sciences. He is particularly interested in hybrid systems, networked embedded systems, and systems biology. 


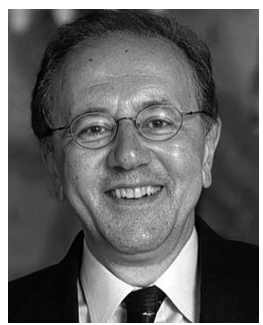

Panos J. Antsaklis (S'74-M'76-SM'86-F'91) is a graduate of the National Technical University of Athens (NTUA), Athens, Greece, and received the M.S. and Ph.D. degrees from Brown University, Providence, RI, in 1974 and 1977, respectively.

$\mathrm{He}$ is the H. Clifford and Evelyn A. Brosey Professor of Electrical Engineering and Concurrent Professor of Computer Science and Engineering at the University of Notre Dame, Notre Dame, IN. He served as the Director of the Center for Applied Mathematics of the University of Notre Dame from 1999 to 2005. His recent research focuses on networked embedded systems and addresses problems in the interdisciplinary research area of control, computing and communication networks, and on hybrid and discrete event dynamical systems. He has authored a number of publications in journals, conference proceedings and books, and he has edited six books on intelligent autonomous control, hybrid systems and on networked embedded control systems. In addition, he has coauthored the research monographs Supervisory Control of
Discrete Event Systems Using Petri Nets (Norwell, MA: Kluwer, 1998) and Supervisory Control of Concurrent Systems: A Petri Net Structural Approach (Boston, MA: Birkhäuser, 2006) and the graduate textbook Linear Systems (New York: McGraw-Hill, 1997).

Dr. Antsaklis has been a Guest Editor of special issues in the IEEE Transactions on Automatic Control and the Proceedings of the IEEE on Hybrid and Networked Control Systems. He serves on the editorial boards of several journals, and he currently serves as an AEAL of the IEEE TRANSACTIONS ON AUTOMATIC CONTROL. He has served as Program Chair and General Chair of major systems and control conferences and he was the 1997 President of the IEEE Control Systems Society (CSS). He has been plenary and keynote speaker in a number of conferences and research workshops. He currently serves as the president of the Mediterranean Control Association. He is a Distinguished Lecturer of the IEEE Control Systems Society, a recipient of the IEEE Distinguished Member Award of the Control Systems Society, and an IEEE Third Millennium Medal recipient. He was the 2006 recipient of the Brown Engineering Alumni Medal of Brown University. 